\title{
Optimal Design of Wireless Networks for Broadband Access with Minimum Power Consumption
}

\author{
Daniel Verenzuela*, Emil Björnson*, Luca Sanguinetti ${ }^{\dagger \ddagger}$ \\ *Department of Electrical Engineering, Linköping University, Linköping, Sweden. \\ †Dipartimento di Ingegneria dell'Informazione, University of Pisa, Pisa, Italy. \\ ${ }_{\ddagger}^{\ddagger}$ Large Networks and System Group (LANEAS), CentraleSupélec, Université Paris-Saclay, Gif-sur-Yvette, France.
}

\begin{abstract}
The continuous rise in wireless data traffic brings forth an increase in power consumption and static users constitute a large fraction of these traffic demands. This work focuses on designing cellular networks to deliver a given data rate per area and user, while minimizing the power consumption. In particular we are interested in optimizing the transmission power, density of access points (APs), number of AP antennas and number of users served in each cell. To this end, we consider a network model based on stochastic geometry and a detailed power consumption model to derive closed form expressions and obtain insights on the interplay of the aforementioned design parameters. The results show that, in contrast with previous works on optimal network design for energy efficiency, having exceedingly high AP density does not bring the most benefits in terms of power savings. Instead the AP density should be chosen according to the area data rate that we want to deliver. In addition numerical results show that the minimum power consumption is obtained in the Massive MIMO regime with many antennas and users per AP.
\end{abstract}

\section{INTRODUCTION}

In the evolution of mobile networks the rise of data traffic continues to be a dominant trend [1] that in turn increases the power consumption of communication networks [2]. There are growing environmental and economic concerns about how the traffic growth can be sustained and the answer seems to be in technological developments to reduce the power consumption [3]. The challenges for new generation technologies involve a growth of $1000 \times$ in area throughput without increasing the power consumption [4], [5]. Therefore increasing the energy efficiency (EE), defined as the ratio between data throughput and energy consumption, has been the focus of much research over the past decade [6].

In general, there are two different approaches for improving EE, namely, small-cell networks [7], [8] and large-scale or massive multiple-input multiple-output (MIMO) systems [9][12]. Both technologies can improve the area throughput and reduce the radiated power, but at the cost of deploying more hardware infrastructure. Hence, the overall EE of the network can only be improved if these benefits and costs are properly balanced. A first attempt in this direction was taken in [13], [14] where the authors developed an EE maximization framework that provides valuable insights on the interplay among the different network parameters. Among others, these include transmission power, density of access points (APs), number of transmit antennas per AP, and number of user equipments (UEs) simultaneously served by each AP. The analysis showed

This paper has received funding from ELLIIT, CENIIT and the Swedish Foundation for Strategic Research. Luca Sanguinetti has been supported by the ERC Starting Grant 305123 MORE and by the research project 5GIOTTO funded by the University of Pisa. that reducing the cell size is undoubtedly the way towards high EE. However, the benefits saturate after a certain point and further improvements can only be obtained through a massive MIMO configuration.

While it is good to have a fundamental understanding of how to design energy-efficient networks, pure EE optimization can lead to solutions with low data throughput or high power consumption. A more practical problem formulation is to design a network that can provide a certain service performance with minimal power consumption. In this context, the work [8] minimizes the power consumption for different AP sleeping techniques in heterogeneous networks. In [15], a comparison between micro and macro cells with distributed and co-located antennas is done in terms of energy consumption. The paper [16] studies the optimal AP density to minimize the area power consumption. Although these works provide interesting insights, a comprehensive analytical network optimization for minimum power consumption is not available in the literature.

In this work we develop a framework that allows to understand how a cellular network designed for minimum power consumption should look like. In a wireless network the spatial distribution of APs is highly irregular and the cell geometry plays a key role when determining the performance. Stochastic geometry is a promising tool to derive tractable analytical results and to model the random properties of practical network deployments [17]. Therefore, we consider the downlink of a network with zero-forcing ( $\mathrm{ZF}$ ) precoding in which the locations of APs are distributed according to a homogeneous Poisson point process (PPP). Furthermore, we adopt the detailed power consumption model developed in [12]. All this is then used to formulate an optimization problem aimed at minimizing the area power consumption (APC) subject to constraints on the individual average data rate $(R)$ of UEs and the area data rate (AR). The optimization is carried out with respect to the AP density, number of AP antennas, number of UEs served per AP and transmission power.

The results show that, in contrast to previous works that optimize the EE, increasing the density of APs indefinitely does not always bring benefits in terms of power savings. In fact, the optimal approach is to select the AP density based on the AR that should be delivered. We derive closed form expressions showing the interplay of main design parameters that provide important insights on how a network with minimum power consumption should be designed. The numerical results show that a massive MIMO configuration with many antennas and UEs per cell is optimal and significantly reduces the density of APs needed to satisfy a given AR requirement 


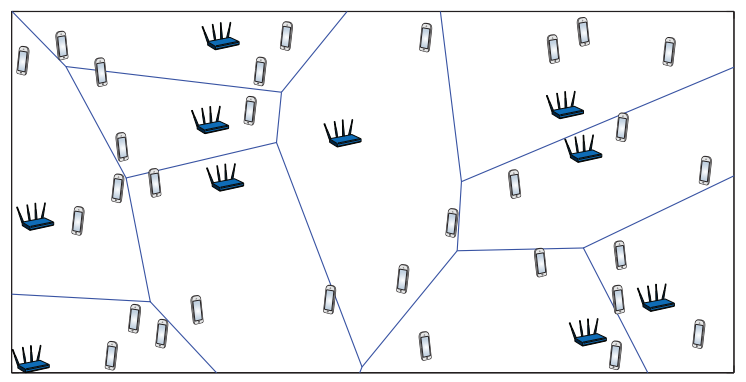

Figure 1. Example of the spatial distribution of APs according to a homogeneous PPP $\Psi_{\lambda}$ with $M=4$ antennas per AP and $K=3$ UEs uniformly distributed within each Voronoi cell.

in comparison to a system with a single UE per cell.

\section{NeTwORK MODEL}

Given that the traffic generated by low mobility indoor UEs (e.g., video streaming) constitutes a vast portion of the power consumption in cellular networks we focus on delivering their service as efficient as possible. Following [14], we consider the downlink of a network operating over a bandwidth $B_{W}$ in which all APs are active and randomly distributed according to a homogeneous PPP $\Psi_{\lambda}$ with intensity $\lambda\left[\mathrm{APs} / \mathrm{km}^{2}\right]$. Each AP is equipped with $M$ antennas and serves $K \leq M$ singleantenna UEs uniformly distributed within the Voronoi cells (see Figure 1). The UEs are assumed to be static and equipped with transceivers affected by hardware impairments [11].

The stationarity of UEs implies that the coherence time is large enough so that the overhead introduced by channel estimation can be neglected. Moreover the effects of hardware impairments in the UEs are considered to be dominant over the channel estimation errors and in turn these are also neglected [11]. We concentrate our analysis on a typical UE with the arbitrary index $k$ connected to AP $0 \in \Psi_{\lambda}$. Such a UE statistically represents any UE in the network by means of the translation invariance property of PPPs. We denote by $\mathbf{h}_{i, k} \in \mathbb{C}^{N}$ the channel vector, at an arbitrary subcarrier, between AP $i \in \Psi_{\lambda}$ and the typical UE $k$ and assume that

$$
\mathbf{h}_{i, k} \sim \mathcal{C N}\left(\mathbf{0}, \omega^{-1} d_{i, k}^{-\alpha} \mathbf{I}_{M}\right)
$$

where $\alpha>2$ is the path loss exponent, $d_{i, k}[\mathrm{~km}]$ denotes the distance between AP $i$ and UE $k$ and $\omega$ determines the average propagation loss (including wall penetrations) at a reference distance of $1 \mathrm{~km}$. We assume ZF precoding and denote $\mathbf{W}_{i}=\left[\mathbf{w}_{i, 1} \ldots \mathbf{w}_{i, K}\right] \in \mathbb{C}^{M \times K}$ as the precoding matrix of AP $i$ with $\mathbf{w}_{i, k}$ being the normalized precoding vector associated to UE $k$ and given by

$$
\mathbf{w}_{i, k}=\frac{\mathbf{H}_{i}\left(\mathbf{H}_{i}^{H} \mathbf{H}_{i}\right)^{-1} \mathbf{e}_{k}}{\left\|\mathbf{H}_{i}\left(\mathbf{H}_{i}^{H} \mathbf{H}_{i}\right)^{-1} \mathbf{e}_{k}\right\|}
$$

where $\mathbf{H}_{i}=\left[\mathbf{h}_{i, 1} \ldots \mathbf{h}_{i, K}\right] \in \mathbb{C}^{M \times K}$ is the channel gain matrix between AP $i$ and its associated $K$ UEs, $\mathbf{e}_{k}$ denotes the $k$ th column of the identity matrix and the notation $\|\cdot\|$ stands for the Euclidean norm. Notice that for $\mathbf{W}_{i}$ to exist the matrix $\mathbf{H}_{i}^{H} \mathbf{H}_{i}$ needs to be invertible. This is why we need to enforce the condition $M \geq K$.
Let $\mathbf{s}_{i} \sim \mathcal{C N}\left(\mathbf{0}, \mathbf{I}_{K}\right)$ be the vector collecting the normalized data symbols sent by AP $i$ to its associated $K$ UEs and call $\rho>0$ the average transmission power per UE over the complete bandwidth $B_{W} \cdot{ }^{1}$ Then, the signal received at the typical UE $k$ is written as

$$
\begin{aligned}
y_{k}= & \sqrt{1-\epsilon^{2}}\left(\sqrt{\frac{\rho}{\mathrm{B}_{\mathrm{w}}}} \mathbf{h}_{0, k}^{H} \mathbf{W}_{0} \mathbf{s}_{0}+\sum_{i \in \Psi_{\lambda} \backslash\{0\}} \sqrt{\frac{\rho}{\mathrm{B}_{\mathrm{w}}}} \mathbf{h}_{i, k}^{H} \mathbf{W}_{i} \mathbf{s}_{i}\right) \\
& +r_{k}+n_{k}
\end{aligned}
$$

where $n_{k} \sim \mathcal{C N}\left(0, \frac{\sigma^{2}}{B_{W}}\right)$ with $\sigma^{2}$ being the noise power over the bandwidth $B_{W}$. The impact of hardware impairments is modeled based on [11] as a reduction of the received signal energy of $1-\epsilon^{2}$ and an additive distortion noise given by

$$
r_{k} \sim \mathcal{C N}\left(0, \epsilon^{2} \frac{\rho}{\mathrm{B}_{\mathrm{w}}} \sum_{i \in \Psi_{\lambda}}\left\|\mathbf{h}_{i, k}^{H} \mathbf{W}_{i}\right\|^{2}\right)
$$

where $0<\epsilon<1$ is a parameter related to the error vector magnitude (EVM). The EVM is used to measure the quality of the transceiver defined as the ratio between average distortion magnitude and signal magnitude, which yields $\frac{\epsilon}{\sqrt{1-\epsilon^{2}}} \approx \epsilon$ in our notation. We only consider hardware impairments at the UEs since these are expected to dominate in the future [11].

Lemma 1. A lower bound on the average achievable rate per user [bit/s/user] in the network with $Z F$ processing is given by

$$
\mathrm{R}=\mathrm{B}_{\mathrm{w}} \log _{2}\left(1+\frac{\left(1-\epsilon^{2}\right)(M-K)}{\frac{2 K}{(\alpha-2)}+\epsilon^{2}(M-K)+\frac{\Gamma(\alpha / 2+1)}{(\pi \lambda)^{\alpha / 2}} \frac{\omega \sigma^{2}}{\rho}}\right)
$$

where $M \geq K+1$ and $\Gamma(\cdot)$ is the gamma function.

Proof: The proof can be found in [14, Prop. 1].

Notice that $M=K$ is not supported by this lemma because of the bounding technique used for deriving the lower bound on the capacity. The rate expression in (5) is used in the next section to define our metrics and cost functions in closed form.

\section{PROBlem Formulation}

The goal of this work is to find the network parameters that minimize the APC [Watt $/ \mathrm{km}^{2}$ ] while providing a certain data rate per UE as well as a given average data rate per $\mathrm{km}^{2}$. The latter is denoted as AR $\left[\mathrm{bit} / \mathrm{s} / \mathrm{km}^{2}\right]$ and is defined by

$$
\mathrm{AR}=\lambda K \mathrm{R} .
$$

The APC comprises all the power consumed at the APs and UEs by the transmitted signals, circuit dissipation, transceiver signal processing, backhaul signaling and overhead, such as site cooling. The APC is defined as in [12] and given by

$$
\mathrm{APC}=\lambda\left(\frac{K \rho}{\eta}+\mathcal{C}_{0}+\mathcal{C}_{1} K+\mathcal{D}_{0} M+\mathcal{D}_{1} M K\right)+\mathcal{A} \mathrm{AR}
$$

where $\eta \in(0,1]$ is the efficiency of the power amplifier and $\mathcal{C}_{0}>0$ represents the fixed power consumption at each AP, independent of the load or number of antennas. In addition, the term $\mathcal{C}_{1}>0$ accounts for the power consumed by all

\footnotetext{
${ }^{1}$ For tractability we consider equal power allocation and leave the implementation of power control for future work.
} 
circuit components at each UE whereas $\mathcal{D}_{0}>0$ is related to the power consumption of all circuit components at each AP antenna. The power required for signal processing is given by $\mathcal{D}_{1} M K>0$ where the complexity of $M K$ operations, for example, comes from computing the product $\mathbf{W}_{i} \mathbf{s}_{i}$. The term $\mathcal{A}>0$ accounts for the load dependent power consumption (e.g., coding, decoding, backhaul). The forthcoming analysis holds for any positive values of the above parameters, however some examples are given in Table I. More details on the above power consumption model can be found in [12].

Denote by $\gamma$ the target data rate per UE and by $\xi$ the required area data rate. Thus, the optimization problem is mathematically formulated as:

$$
\begin{aligned}
& \underset{\rho, \lambda \in \mathbb{R}_{+}}{\operatorname{minimize}} \operatorname{APC}(\rho, \lambda, M, K) \\
& \begin{array}{l}
M, K \in \mathbb{Z}_{+} \\
\text {subject to }
\end{array} \quad \mathrm{R} \geq \gamma \quad \text { and } \quad \mathrm{AR} \geq \xi
\end{aligned}
$$

where $\mathbb{R}_{+}$and $\mathbb{Z}_{+}$denote the set of all strictly positive real and integer numbers respectively. Notice that from Lemma 1 it follows that $\lim _{M \rightarrow \infty} \mathrm{R}=B_{W} \log _{2}\left(1 / \epsilon^{2}\right)$. This implies that (8) is feasible if and only if $0<\gamma<B_{W} \log _{2}\left(1 / \epsilon^{2}\right)$.

\section{Minimizing ThE APC}

In the following section we solve the optimization problem (8) analytically using the $\mathrm{R}$ expression from Lemma 1 while providing closed form expressions of all optimization variables that show the interplay of the main parameters in the system.

\section{A. Optimal Transmission Power per UE}

We begin by deriving the optimal transmission power per UE $\rho$ while the other variables in (8) are kept constant.

Lemma 2. Assume $M, K$ and $\lambda$ are given. If the problem (8) is feasible and

$$
\frac{M}{K}>\frac{2\left(2^{\gamma}-1\right)}{\left(1-\epsilon^{2} 2^{\gamma}\right)(\alpha-2)}+1
$$

then the optimal $\rho$ is given by

$$
\rho^{*}=\frac{\frac{\left(2^{\gamma}-1\right)}{\left(1-\epsilon^{2} 2^{\gamma}\right)} \frac{\omega \sigma^{2} \Gamma(\alpha / 2+1)}{(\pi \lambda)^{\alpha / 2}}}{M-K-\frac{\left(2^{\gamma}-1\right)}{\left(1-\epsilon^{2} 2^{\gamma}\right)} \frac{2 K}{(\alpha-2)}} .
$$

Proof: The result easily follows by observing that APC and $\mathrm{R}$ are both monotonically increasing functions of $\rho$. Therefore, the optimal $\rho^{*}$ is given by the lowest value of $\rho$ for which the data rate constraint per UE is satisfied with equality, i.e., $\mathrm{R}\left(\rho^{*}\right)=\gamma$. Solving the equality with respect to $\rho^{*}$ yields the result. Notice that the condition (9) is required to ensure that $\rho^{*}>0$ and is obtained by setting the denominator of $\rho^{*}$ greater than zero.

We can see that $\rho^{*}$ is a decreasing function of $\lambda$ and $M$ while it increases with $K$. Observe that the same $\rho$-value was obtained in [14, Th. 2] for the problem of EE maximization.
By substituting $\rho^{*}$ into (7) the problem (8) reduces to:

$$
\begin{array}{ll}
\underset{\substack{\lambda \in \mathbb{R}_{+} \\
M, K \in \mathbb{Z}_{+}}}{\operatorname{minimize}} & b \lambda^{-\left(\frac{\alpha}{2}-1\right)}+d \lambda \\
\text { subject to } & \frac{M}{K}>\frac{2\left(2^{\gamma}-1\right)}{\left(1-\epsilon^{2} 2^{\gamma}\right)(\alpha-2)}+1 \\
& \lambda K \geq \frac{\xi}{\gamma}
\end{array}
$$

where

$$
\begin{aligned}
b & =\frac{\omega \sigma^{2} \Gamma(\alpha / 2+1)}{\eta \pi^{\alpha / 2}\left(\left(\frac{M}{K}-1\right)\left(\frac{1-\epsilon^{2} 2^{\gamma}}{2 \gamma-1}\right)-\frac{2}{(\alpha-2)}\right)} \\
d & =\mathcal{C}_{0}+\mathcal{C}_{1} K+\mathcal{D}_{0} M+\mathcal{D}_{1} M K+\mathcal{A} K \gamma
\end{aligned}
$$

The inequality (13) comes from the definition of the AR in (6) and the fact that $\mathrm{R}\left(\rho^{*}\right)=\gamma$. For later convenience, from the condition in (12) we define

$$
c_{\min }=\frac{2\left(2^{\gamma}-1\right)}{\left(1-\epsilon^{2} 2^{\gamma}\right)(\alpha-2)}+1
$$

as the limiting ratio between $M$ and $K$ such that $\rho^{*}>0$. Notice that $c_{\mathrm{min}}$ is an increasing function of $\gamma$, meaning that a larger number of AP antennas is needed if the rate constraint per UE grows.

\section{B. Optimal AP Density}

Now we find the optimal $\lambda$ in (11) when $M$ and $K$ are given.

Theorem 1. Suppose that $M$ and $K$ are fixed at some arbitrary values for which (11) is feasible, then the optimal $\lambda$ is

$$
\lambda^{*}=\max \left\{\hat{\lambda}, \frac{\xi}{\gamma K}\right\}
$$

with

$$
\hat{\lambda}=\left(\frac{b}{d}\left(\frac{\alpha}{2}-1\right)\right)^{\frac{2}{\alpha}}
$$

Proof: The result easily follows by observing that the objective function $f=b \lambda^{-(\alpha / 2-1)}+d \lambda$ in (11) is convex for $\alpha>2$ and that $\lim _{\lambda \rightarrow 0} f=\infty$ and $\lim _{\lambda \rightarrow \infty} f=\infty$. Therefore, there exists a value of $\lambda$ for which $\partial f / \partial \lambda=0$. This yields $\hat{\lambda}$. From (13), it also follows that $\lambda$ must satisfy $\lambda \geq \frac{\xi}{\gamma K}$. Putting these facts together concludes the proof.

From Theorem 1, it follows that if the AR constraint is removed (i.e., $\xi=0$ ), then the optimal $\lambda$ is always given by $\hat{\lambda}$ in (17) irrespective of the rate constraint per UE $\gamma$. As shown in (17) $\hat{\lambda}$ is monotonically decreasing with $M$ and with all the terms of the power consumption model (i.e., $\eta, \mathcal{C}_{0}, \mathcal{C}_{1}, \mathcal{D}_{0}$, $\mathcal{D}_{1}$ and $\mathcal{A}$ ). Furthermore, if the ratio $M / K$ is kept fixed, then $\hat{\lambda}$ is also monotonically decreasing with $K$. Therefore, if no AR constraint is imposed and practical values of $M$ and $K$ are considered, the optimal density can be very small (see for example the results illustrated in Figure 2) and the resulting solution will provide poor AR, thus making the system unable to support the rising demand for higher area data rates. On the other hand, for $\xi$ values of practical interest such that $\xi \geq \xi_{\min }$ with $\xi_{\min }=\hat{\lambda} \gamma K$, then the AP density that minimizes the 
power consumption is exclusively determined by the area data rate constraint and is given by

$$
\lambda^{*}=\frac{\xi}{\gamma K}
$$

This is in sharp contrast to the results for the EE maximization problem (e.g., [13], [14]) wherein the EE is a monotonically increasing function of the AP density and thus it is preferable to have as high AP density as possible. From (18), it follows that $\lambda^{*}$ depends linearly on the target average area data rate constraint $\xi$ and it is inversely proportional to the number $K$ of UEs per AP. In the following analysis, we only consider the practically most interesting case for which $\xi$ is large enough such that the constraint $\xi \geq \xi_{\min }$ is always satisfied.

\section{Optimal Number of UEs per AP}

Next, we want to find the optimal $K$. To this end, we substitute $\lambda^{*}=\frac{\xi}{\gamma K}$ into (11) and obtain:

$$
\begin{aligned}
& \underset{M, K \in \mathbb{Z}_{+}}{\operatorname{minimize}} \\
& \frac{\hat{b}\left(\frac{\xi}{\gamma K}\right)^{-\left(\frac{\alpha}{2}-1\right)}}{\left(\frac{M}{K}-1\right) \hat{d}-\frac{2}{(\alpha-2)}}+\frac{\xi}{\gamma}\left(\frac{\mathcal{C}_{0}}{K}+\mathcal{C}_{1}+\mathcal{D}_{0} \frac{M}{K}+\mathcal{D}_{1} \frac{M}{K} K+\mathcal{A} \gamma\right), \\
& \text { subject to } \quad \frac{M}{K}>c_{\text {min }},
\end{aligned}
$$

where

$$
\hat{b}=\frac{\omega \sigma^{2} \Gamma(\alpha / 2+1)}{\eta \pi^{\alpha / 2}} \quad \text { and } \quad \hat{d}=\left(\frac{1-\epsilon^{2} 2^{\gamma}}{2^{\gamma}-1}\right) .
$$

Notice that the objective function of (19) grows large as $M, K \rightarrow \infty$ thus the optimal solution must be at finite integer values of $M$ and $K$ that can be easily found by using an exhaustive search algorithm. However, we also want to understand the structure of the solution, which cannot be obtained from a numeric search algorithm, and therefore we relax the integer constraints to find the best real-valued solutions. Moreover since $M$ is dependent on $K$ through the inequality in (20), we denote the number of antennas per UE as $\bar{c}=\frac{M}{K}$ and look for the optimal $K$ under the assumption that $\bar{c}$ is given.

Theorem 2. Consider any fixed positive $\bar{c}>c_{\min }$ for which the problem (19) is feasible, then the optimal number of UES per cell is computed as

$$
K^{*}=K^{(o)}
$$

where the value $\left\{K^{(o)}\right\}$ is the real positive solution of

$$
\frac{\hat{b}\left(\frac{\xi}{\gamma}\right)^{-\frac{\alpha}{2}}\left(\frac{\alpha}{2}-1\right)}{\left((\bar{c}-1) \hat{d}-\frac{2}{(\alpha-2)}\right)} K^{\alpha / 2}+\mathcal{D}_{1} \bar{c} K^{2}-\mathcal{C}_{0}=0 .
$$

Proof: The objective function of (19), denoted by $g$, is convex in $K$ for $\alpha \geq 4$ whereas it is quasi-convex for $2<\alpha<4$ and both have their global minimum located at $K^{(o)}>0$ where $\frac{\partial g}{\partial K}=0$. When $\alpha \geq 4$ the proof of convexity is straightforward by evaluating the second derivative condition with $K>0$. When $2<\alpha<4$, we have that $g \rightarrow \infty$ as $K \rightarrow 0$ and that $\partial^{2} g / \partial K^{2}>0$ if

$$
K<K_{\max }=\frac{\xi}{\gamma}\left(\frac{\mathcal{C}_{0}\left((\bar{c}-1) \hat{d}-\frac{2}{(\alpha-2)}\right)}{\hat{b}\left(\frac{\alpha}{2}-1\right)\left(1-\frac{\alpha}{4}\right)}\right)^{2 / \alpha}
$$

whereas $\partial g / \partial K>0$ if

$$
K>K_{\min }=\frac{\xi}{\gamma}\left(\frac{\mathcal{C}_{0}\left((\bar{c}-1) \hat{d}-\frac{2}{(\alpha-2)}\right)}{\hat{b}\left(\frac{\alpha}{2}-1\right)}\right)^{2 / \alpha} .
$$

Thus $g$ is convex for $0<K<K_{\max }$ and it is also monotonically increasing for $K \geq K_{\max }$ since $K_{\min }<K_{\max }$ (i.e., $g$ is a quasi-convex function). This means that the global minimum lies in the interval $\left(0, K_{\max }\right)$ and thus it is achieved for the $K^{(o)}>0$ such that $\partial g / \partial K=0$.

Notice that finding the solution of (23) is easy since the problem is convex with respect to $K$ within the range of interest. However, to obtain better insights on the fundamental trade-offs between the parameters that define the optimal number of UEs per cell, let us look at the special case of $\alpha=4$ which is a common approximation for the path loss exponent and makes (23) a second order polynomial equation.

Corollary 1. Consider the path loss exponent $\alpha=4$ and suppose the optimization problem in (19) is feasible, then the optimal $K$ for any given value of $\bar{c}>c_{\min }$ is

$$
K^{*}=\sqrt{\frac{\mathcal{C}_{0}((\bar{c}-1) \hat{d}-1)}{\hat{b}\left(\frac{\gamma}{\xi}\right)^{2}+\mathcal{D}_{1} \bar{c}((\bar{c}-1) \hat{d}-1)}} .
$$

From (26) we see that when $\alpha=4$ the optimal $K^{*}$ is monotonically increasing with $\mathcal{C}_{0}$ and monotonically decreasing with $\mathcal{D}_{1}$. This means that as the fixed power consumption decreases less UEs need to be served in each cell to minimize the APC. Also if the power consumption corresponding to signal processing tasks decreases, then more UEs can be served. We see that the optimal $K^{*}$ remains unaffected by the parameters $\mathcal{D}_{0}$ and $\mathcal{C}_{1}$.

\section{Optimal Number of AP Antennas}

Finally, let us optimize the number of AP antennas $M$ while $K$ is kept constant.

Theorem 3. Consider $K$ to be given and the optimization problem in (19) to be feasible, then the optimal number of AP antennas is obtained as

$$
M^{*}=\bar{c}^{*} K
$$

where

$$
\bar{c}^{*}=\left(\frac{\hat{b}\left(\frac{\xi}{\gamma}\right)^{-\frac{\alpha}{2}} K^{\left(\frac{\alpha}{2}-1\right)}}{\hat{d}\left(\mathcal{D}_{0}+\mathcal{D}_{1} K\right)}\right)^{1 / 2}+\frac{2}{\hat{d}(\alpha-2)}+1
$$

Proof: The objective function of (19), denoted by $g$, is a convex function of $\bar{c}$ for $\bar{c}>\bar{c}_{\text {min }}$ and the global minimum is found when $\frac{\partial g}{\partial \bar{c}}=0$. 
Table I. Simulation PARAmeters

\begin{tabular}{|c|c|c|}
\hline Parameter & Symbol & Value \\
\hline Path loss exponent & $\alpha$ & 3.76 \\
\hline Fixed propagation loss $(1 \mathrm{~km})$ & $\omega$ & $130 \mathrm{~dB}$ \\
\hline Power amplifier efficiency & $\eta$ & 0.39 \\
\hline Level of hardware impairments & $\epsilon$ & 0.05 \\
\hline System bandwidth & $\mathrm{B}_{\mathrm{W}}$ & $20 \mathrm{MHz}$ \\
\hline Load power consumption & $\mathcal{A ~}_{\mathrm{W}}$ & $0.023 \mathrm{~W} / \mathrm{bit}$ \\
\hline Static power consumption & $\mathcal{C}_{0}$ & $10 \mathrm{~W}$ \\
\hline Circuit power per active UE & $\mathcal{C}_{1}$ & $0.1 \mathrm{~W}$ \\
\hline Circuit power per AP antenna & $\mathcal{D}_{0}$ & $1 \mathrm{~W}$ \\
\hline Signal processing coefficient & $\mathcal{D}_{1}$ & $3.12 \mathrm{~mW}$ \\
\hline Noise variance over system bandwidth & $\sigma^{2}$ & $2 \cdot 10^{-13} \mathrm{~W}$ \\
\hline
\end{tabular}

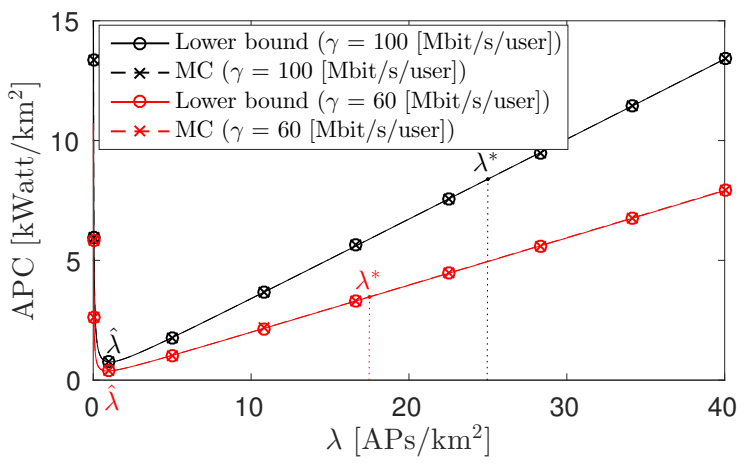

Figure 2. Area power consumption $\left[\mathrm{kWatt} / \mathrm{km}^{2}\right]$ as a function of $\lambda$ for $\xi=20\left[\mathrm{Gbit} / \mathrm{s} / \mathrm{km}^{2}\right]$. The vertical lines depict the optimal $\lambda^{*}$ (from (16)). The minimum values found in Theorem 1 are depicted as $\hat{\lambda}$. The values of $\rho$, $M$ and $K$ are optimized to yield minimum APC.

Notice that $\bar{c}^{*}$ decreases monotonically with $\mathcal{D}_{0}$ and $\mathcal{D}_{1}$, which means that as the circuit power and signal processing power per AP antenna decrease a larger number of antennas can be used for minimizing the APC. Furthermore, from (23) we see that $K^{*}$ decreases as $\mathcal{C}_{0}$ grows large and thus the same occurs for $M^{*}$. On the other hand, the term $\mathcal{C}_{1}$ does not affect the optimal number of AP antennas.

\section{Simulation Results}

This section validates and illustrates the analytical insights from Section IV by a numerical study. The selection of main parameters is listed in Table I and is based on [12], [14].

First we would like to show how the APC depends on the density of APs to see the impact of deploying denser networks. Figure 2 shows the APC as a function of $\lambda$ for two different values of $\gamma$ when the optimal $\rho$ and the optimal integer-valued choice of $M$ and $K$ are used. Notice that the APC is a convex function and the minimum lies at low values of $\lambda$, which validates the insights given by Theorem 1 . This means that, for most practical AP densities, the APC is an increasing function of $\lambda$, in fact we can see that the optimal values of $\lambda^{*}$ (for $\gamma=\{60,100\}[\mathrm{Mbit} / \mathrm{s} / \mathrm{user}]$ ) that guarantee an AR of $\xi=20\left[\mathrm{Gbit} / \mathrm{s} / \mathrm{km}^{2}\right]$ are well above the minimum. Note that this particular value of $\xi$ is conservative when compared to the future values 170 and $10^{5}\left[\mathrm{Gbit} / \mathrm{s} / \mathrm{km}^{2}\right]$ predicted by the METIS project [18] in shopping malls and virtual reality office scenarios, respectively. Thus, increasing the density of APs without bound is not the best approach to design a network with minimum power consumption. Moreover, observe that the APC increases as $\gamma$ grows large and this effect increases as $\lambda$ takes larger values. It is also worth mentioning that the lower bound on $\mathrm{R}$ provided in Lemma 1 yields practically

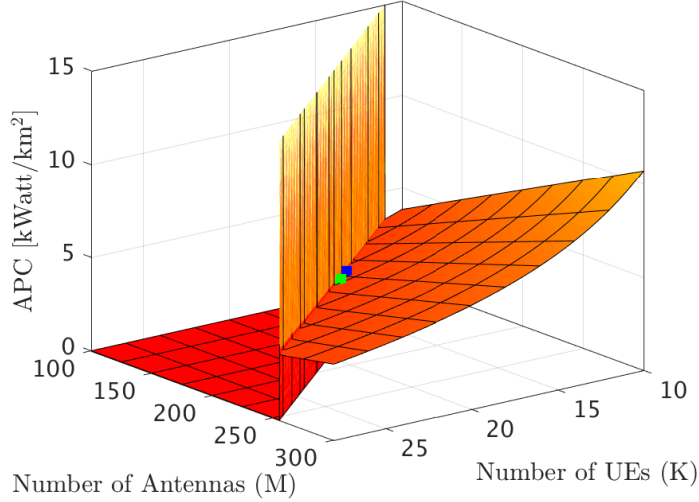

(a) 3D graph of the APC vs $M$ and $K$, with optimal real-value (blue square where $M=165.35$ and $K=18.06$ ) and exhaustive search integer (green square where $M=174$ and $K=19$ ) solutions.
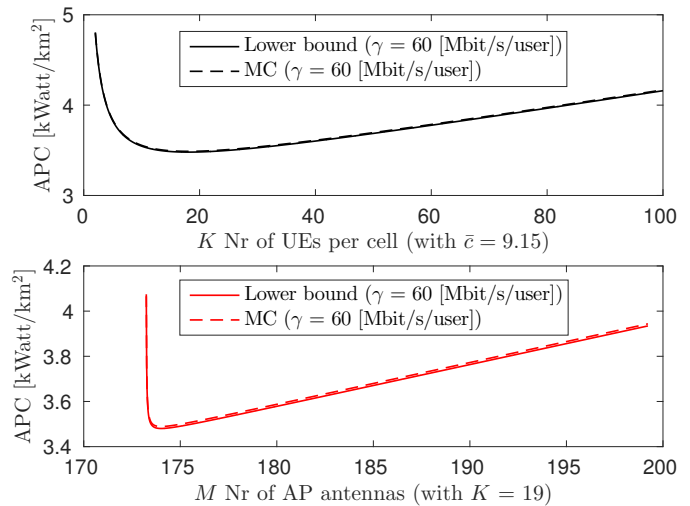

(b) APC as a function of $K$ for a fixed $\bar{c}=9.15$ (top graph) and as a function of $M$ for a fixed $K=19$ (bottom graph).

Figure 3. Area power consumption $\left[\mathrm{kWatt} / \mathrm{km}^{2}\right]$ vs $M$ and $K$ for $\gamma=60$ [Mbit/s/user] and $\xi=20\left[\mathrm{Gbit} / \mathrm{s} / \mathrm{km}^{2}\right]$. The results are given for optimal values of $\rho$ and $\lambda$.

the same results for the APC as the Monte-Carlo simulations, which validates the tightness of this analytically tractable lower bound.

Figure 3(a) depicts the APC as a function of both $M$ and $K$ for $\gamma=60[\mathrm{Mbit} / \mathrm{s} / \mathrm{user}]$ with optimal values of $\lambda$ and $\rho$. We can see that the exhaustive search algorithm yields the optimal solution $\left(M^{*}, K^{*}\right)=(174,19)$ with a total transmission power per cell of $K^{*} \rho^{*}=302 \mathrm{~mW}$ which corresponds to a Massive MIMO setup wherein a large number of AP antennas is used to serve a smaller number of UEs. Notice that the global minimum APC from the integer and real-valued solutions differ only by $0.01 \%$ due to rounding effects. To illustrate the convex shape of the APC, Figure 3(b) depicts its value as a function of $K$ for a fixed $\bar{c}$ and as a function of $M$ for a fixed $K$. Notice that the particular fixed values of $\bar{c}$ and $K$ correspond to the optimal points. Also, the results of 3(b) confirm the tightness of the lower bound given by Lemma 1 .

To provide some insights on the impact that the desired $\mathrm{AR}$ has on the network design, we illustrate the behavior of the APC and the optimal AP density versus $\xi$. Figure 4 depicts the APC for optimal values of $\lambda$ and $\rho$ comparing 


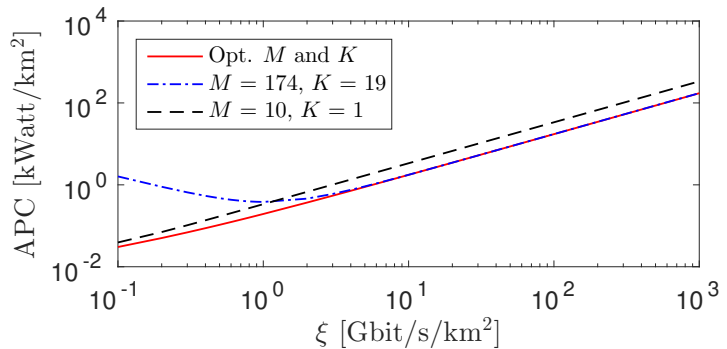

Figure 4. Area power consumption $\left[\mathrm{kWatt} / \mathrm{km}^{2}\right]$ as a function of $\xi$ for $\gamma=60$ [Mbit/s/user]. The values of $\rho$ and $\lambda$ are chosen as optimal.

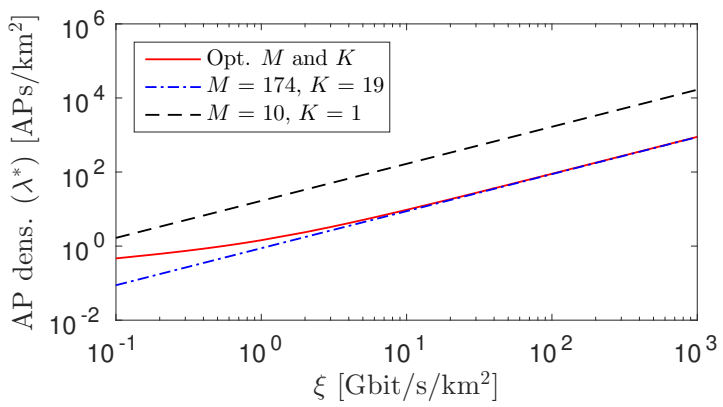

Figure 5. Density of APs $\left[\mathrm{AP} / \mathrm{km}^{2}\right]$ as a function of $\xi$ for $\gamma=60$ [Mbit/s/user]. The choice of $\rho$ is optimal.

three network configurations: an optimal choice of $M$ and $K$, a fixed massive MIMO (i.e., $M=174, K=19$ ) and a single UE per cell. Figure 5 shows the optimal density of APs as a function of $\xi$ for an optimal $\rho$ comparing the same configurations. We can see that both the APC and the density of APs are increasing functions of the AR with the only exception of the APC for the fixed Massive MIMO setup. In this case, the APC has a convex shape and for low values of $\xi$ the APC takes larger values than with a single UE per cell system. This means that massive MIMO is only desirable if $\xi$ is sufficiently high, otherwise it is better to use smaller cells with fewer UEs. However, we can see that the APC of the Massive MIMO setup converges to the optimal behavior for values of $\xi$ above $10\left[\mathrm{Gbit} / \mathrm{s} / \mathrm{km}^{2}\right]$, which is well below the $\mathrm{AR}$ requirements of future networks estimated by the METIS project [18] mentioned earlier. Notice also that for $\xi$ above $10\left[\mathrm{Gbit} / \mathrm{s} / \mathrm{km}^{2}\right]$ both the optimal and Massive MIMO configurations offer a reduction of $2 \times$ power consumption while needing $19 \times$ less AP density approximately. The latter fact could greatly simplify the practical network deployment.

\section{CONCLUSION}

In this paper, we studied the optimal design of cellular networks for high requirements on the average data rate per area and UEs while having minimal power consumption. This was done by formulating an optimization problem using stochastic network geometry and realistic power consumption models. The problem was solved analytically by deriving closed form expressions for the optimization variables: the transmission power per UE, density of APs, number of UEs per cell and number of AP antennas.

The results showed that, in contrast to previous works [13], [14] on EE optimization, increasing the density of APs without bound is not the proper way to go in order to design practical networks with minimal power consumption. Instead, the AP density must be fixed on the basis of the AR requirement and a massive MIMO setup must be deployed. This implementation significantly reduces the power consumption and AP density by multiplexing several UEs simultaneously with the use of a large number of AP antennas to mitigate interference.

This work assumes static indoor users, in future work we will extend the model to cover user mobility scenarios where channel estimation errors and overhead cannot be neglected.

\section{REFERENCES}

[1] Cisco, "Visual networking index: Global mobile data traffic forecast update, 2014-2019," Tech. Rep., Feb. 2015.

[2] W. Van Heddeghem, S. Lambert, B. Lannoo, D. Colle, M. Pickavet, and P. Demeester, "Trends in worldwide ICT electricity consumption from 2007 to 2012," in Computer Commun., vol. 50, pp. 64-76, 2014.

[3] A. Fehske, G. Fettweis, J. Malmodin and G.Biczok, "The global footprint of mobile communications: The ecological and economic perspective," in IEEE Trans. Commun., vol. 49, no. 8, pp. 55-62, 2011.

[4] GreenTouch Green Meter Research Study, "Reducing the net energy consumption in communications networks by up to $90 \%$ by 2020 ," Tech. Rep., Jun. 2013.

[5] J.G. Andrews, S. Buzzi, W. Choi, S.V. Hanly, A. Lozano, A.C.K. Soong and J.C. Zhang, "What Will 5G Be?,"IEEE J. Sel. Areas Commun., vol.32, no.6, pp. 1065-1082, 2014.

[6] D. Feng, C. Jiang, L. Gubong, L.J. Cimini Jr., G. Feng and G.Y. Li, "A survey of energy-efficient wireless communications," IEEE J. Commun. Surveys \& Tutorials, vol.15, no.1, pp. 167-178, 2013.

[7] J. Hoydis, M. Kobayashi and M. Debbah, "Green Small-Cell Networks," IEEE Veh. Technol. Mag., vol.6, no.1, pp. 37-43, 2011.

[8] Y. S. Soh, T.Q.S. Quek, M. Kountouris and H. Shin, "Energy Efficient Heterogeneous Cellular Networks," IEEE J. Sel. Areas Commun., vol.31, no.5, pp. 840-850, 2013.

[9] T.L. Marzetta, "Noncooperative Cellular Wireless with Unlimited Numbers of Base Station Antennas," IEEE Trans. Wireless Commun., vol.9, no.11, pp. 3590-3600, 2010.

[10] H. Q. Ngo, E.G. Larsson and T.L. Marzetta, "Energy and Spectral Efficiency of Very Large Multiuser MIMO Systems," IEEE Trans. Commun., vol.61, no.4, pp. 1436-1449, 2013.

[11] E. Björnson, J. Hoydis, M. Kountouris and M. Debbah, "Massive MIMO Systems With Non-Ideal Hardware: Energy Efficiency, Estimation, and Capacity Limits," IEEE Trans. Information Theory, vol.60, no.11, pp. 7112-7139, 2014.

[12] E. Björnson, L. Sanguinetti, J. Hoydis and M. Debbah, "Optimal Design of Energy-Efficient Multi-User MIMO Systems: Is Massive MIMO the Answer?," IEEE Trans. Wireless Commun., vol.14, no.6, pp. 3059-3075, 2015.

[13] E. Björnson, L. Sanguinetti and M. Kountouris, "Deploying Dense Networks for Maximal Energy Efficiency: Small Cells Meet Massive MIMO," to appear IEEE J. Sel. Areas Commun. [Online] Available: http://arxiv.org/abs/1505.01181

[14] E. Björnson, L. Sanguinetti and M. Kountouris, "Designing Wireless Broadband Access for Energy Efficiency: Are Small Cells the Only Answer?," in Proc. IEEE Int. Conf. on Commun. Workshop (ICCW), pp. 136-141, 2015.

[15] E. Kurniawan and A. Goldsmith, "Optimizing cellular network architectures to minimize energy consumption," in Proc. IEEE Int. Conf. Commun. (ICC), pp. 4771-4775, 2012.

[16] S. Sarkar, R.K. Ganti and M. Haenggi, "Optimal base station density for power efficiency in cellular networks," in Proc. IEEE Int. Conf. Commun. (ICC), pp. 4054-4059, 2014.

[17] M. Haenggi, J.G. Andrews, F. Baccelli, O. Dousse and M. Franceschetti, "Stochastic geometry and random graphs for the analysis and design of wireless networks," IEEE J. Sel. Areas Commun., vol.27, no.7, pp. 10291046, 2009.

[18] M. Fallgren, B. Timus, et la., D1.1: Scenarios, requirements and KPIs for 5G mobile and wireless system, ICT-317669-METIS, 2013. 